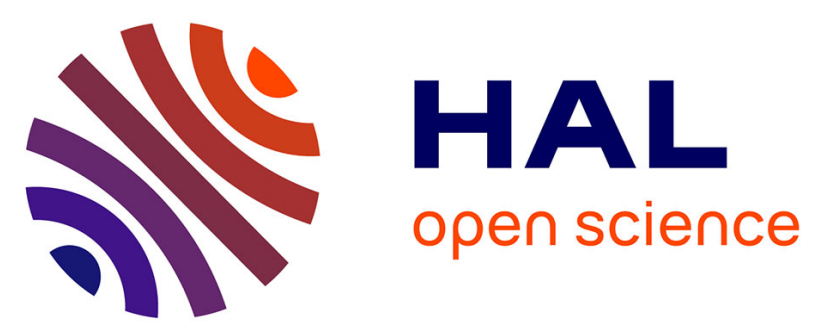

\title{
Mercury speciation in liquid petroleum product : comparison between on-site approach and lab measurement using size exclusion chromatography with high resolution inductively coupled plasma mass spectrometric detection (SEC-ICP-HR MS).
}

Florine Gaulier, Alexandre Gibert, David Walls, Michael Langford, Stuart Baker, Arnaud Baudot, Fabien Porcheron, Charles-Philippe Lienmann

\section{- To cite this version:}

Florine Gaulier, Alexandre Gibert, David Walls, Michael Langford, Stuart Baker, et al.. Mercury speciation in liquid petroleum product: comparison between on-site approach and lab measurement using size exclusion chromatography with high resolution inductively coupled plasma mass spectrometric detection (SEC-ICP-HR MS).. Fuel Processing Technology, 2015, 131, pp.254-261. 10.1016/j.fuproc.2014.10.024 . hal-01128692

\author{
HAL Id: hal-01128692 \\ https://hal.science/hal-01128692
}

Submitted on 10 Mar 2015

HAL is a multi-disciplinary open access archive for the deposit and dissemination of scientific research documents, whether they are published or not. The documents may come from teaching and research institutions in France or abroad, or from public or private research centers.
L'archive ouverte pluridisciplinaire HAL, est destinée au dépôt et à la diffusion de documents scientifiques de niveau recherche, publiés ou non, émanant des établissements d'enseignement et de recherche français ou étrangers, des laboratoires publics ou privés. 
Manuscript Number:

Title: Mercury speciation in liquid petroleum product: Various analytical approaches and comparison

Article Type: Original Research Paper

Keywords: mercury; speciation; ,ICP/MS; Hg; liquid petroleum

Corresponding Author: Dr. Charles-Philippe Lienmann, Ph.D.

Corresponding Author's Institution:

First Author: Charles-Philippe Lienmann, Ph.D.

Order of Authors: Charles-Philippe Lienmann, Ph.D.; Florine Gaulier; Alexandre Gibert; David Walls;

Michael Langford; Stuart Baker; Arnaud Baudot; Fabien Porcheron

Suggested Reviewers: Eva Krupp

e.krupp@abdn.ac.uk

Nicolas Bloom

kolyabloom@gmail.com

Christiane Duyck

cbduyck@vm.uff.br

Maria Ines Monteiro

mmonteiro@cetem.gov.br

Jose Luis Todoli

jose.todoli@ua.es 
3 Florine Gaulier ${ }^{1,3}$, Alexandre Gibert ${ }^{1}$, David Walls ${ }^{2}$, Michael Langford ${ }^{3}$, Stuart Baker $^{2}$, 4 Arnaud Baudot ${ }^{1}$, Fabien Porcheron ${ }^{1,4}$, Charles-Philippe Lienemann ${ }^{1}$

$5 \quad$ IFP Energies Nouvelles-Lyon, Rond-point de l'échangeur de Solaize, BP 3,69360 Solaize, France

$6{ }^{2} \mathrm{Qa}^{3}$, Unit 4, The Calvert Centre, Woodmancott, Winchester, SO21 3BN, United Kingdom

$7 \quad{ }^{3}$ Expro ADS, Chandlers Ford, Hampshire SO53 4DR, United Kingdom

\section{Mercury speciation in liquid petroleum product: Various analytical}

\section{approaches and comparison}

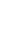

\section{ABSTRACT}

Mercury is a natural contaminant commonly found in liquid hydrocarbon feeds that are processed in the refining and petrochemical industries. It is responsible in particular for catalysts poisoning as well as corrosion of aluminium alloys used in steam cracker cold boxes. Prerequisite for mercury risk assessment relies thus in accurate measurements of mercury content. Moreover, mercury speciation is also crucial in order to propose adequate scavenging solutions aiming at the complete removal of mercury from hydrocarbon streams.

This article is proposing two different approaches to provide accurate speciation of $\mathrm{Hg}$ in liquid fuels. The comparison of a conventional speciation approach - based on filtration, purge and extraction- with hyphenated technique was first carried out on model mercury molecules. A critical assessment of both methods is provided, and comparison of the result obtained with model molecule shows a good validation of the two procedures. Comparison of the two speciation schemes was then performed with real hydrocarbon feeds produced in various locations of the world and complementary information are obtained with chemical properties and size of the $\mathrm{Hg}$ molecules. 


\section{INTRODUCTION}

Mercury $(\mathrm{Hg})$ is a natural contaminant found in earth's crust where its concentration can range from 10 to $20000 \mathrm{ppb}$. Mercury is thus released into the environment from a variety of natural sources, including volcanic, geothermal activities or wildfires; but also from anthropogenic activities. A total of approximately 2000 metric ton of mercury is estimated to be released each year from fossil fuel combustion or metal production [1]. For instance, natural gas production frequently generates hydrocarbon streams containing traces level of mercury [2], especially in Southeast of Asia where $\mathrm{Hg}$ concentration can reach up to $300 \mathrm{ppb}$. Though these levels are rather low, impact on industrial equipment and human health can be serious [3-4]. For instance, mercury has a strong ability to form amalgams with Al-based alloys used in LNG (Liquefied Natural Gas) cryogenic exchangers leading to corrosion issues and potential industrial disaster like the one encountered in 1973 in Skikda, Algeria [5]. In addition, mercury is harmful for human health and numerous incentives have been issued to control and limit its emission from anthropogenic sources[1].

It is well known that in hydrocarbon gas phases, mercury is mainly found as its elemental form $\left(\mathrm{Hg}^{\circ}\right)$ which is highly volatile [6]. However, the speciation of mercury in hydrocarbon liquids continues to be debated in literature. Most of the authors [7-9] are mentioning mainly $\mathrm{Hg}^{\circ}$ and $\mathrm{Hg}(\mathrm{II})$, potentially stabilized by ligands in organic phase, as the main species found in most liquid petroleum cuts. However some uncertainties are suspected due to differences in the main species found. Variation of results lay in mercury species instability over time and uncertainty associated to analytical techniques due to improper validation.

Anyhow, most of the existing mercury removal processes are based on elemental $\mathrm{Hg}$ scavenging by guard beds [10], speciation is thus crucial to assure a complete elimination of mercury from hydrocarbon streams. Many publications are available since the 1990's based on various approaches designed to speciate $\mathrm{Hg}$ in liquid organic stream. A review dedicated 
1 to the petroleum industry was proposed in 2000 by Bouyssiere et al [11] and a more recent

2 and global review on the determination and speciation of $\mathrm{Hg}$ by various analytical techniques

3 [12] focused mainly on environmental samples. For organic substances, nothing has been

4 proposed since 2000 according to our knowledge. Three main approaches can be

5 distinguished.

6 A conventional procedure is based on fractionation of $\mathrm{Hg}$ species by their physico-chemical 7 properties (filtration, purge and extraction). Furuta et al [13] have initiated the studies and was 8 followed by N. Bloom [14], M. Wilhelm [3] and W. Frech [15].

9 A second approach is based on GC coupled with specific detection systems : microwave 10 induced plasma coupled to atomic emission spectrometry (MIP-AES) [9], atomic 11 fluorescence [10] and inductively coupled plasma combined with mass spectrometry 12 (ICP/MS) [7, 8, 16]. Numerous hydrocarbons coelutions are observed provoking high background interferences or loss of signals due to plasma weakness with MIP-AES and, to a lesser extent, with ICP/MS. Moreover, some $\mathrm{Hg}$ compounds are not volatile and require derivatization for complete elution with GC columns, loss of information on initial form of the $\mathrm{Hg}$ compounds is then observed and is limiting for petroleum industry. Furthermore, precautions might be applied due to potential change of $\mathrm{Hg}$ compounds speciation with intensive warming-up procedure. Injection temperature proposed by Bouyssiere [8] $\left(250^{\circ} \mathrm{C}\right)$ and Tao [7] $\left(280^{\circ} \mathrm{C}\right)$ might be too high and therefore lead to apparition of elemental $\mathrm{Hg}$ not initially present.

21 A third category of approach with $\mathrm{Hg}$ speciation is based on liquid chromatography (HPLC) coupled with specific detection systems. Reversed phase HPLC coupled with cold vapor atomic absorption spectroscopy (CVAAS) was initiated by Schickling and Broekaerst in 1995

24 [17]. Difficulties due to heavy organic compounds in CVAAS were partially solved by 25 elimination of carbon matrix with $\mathrm{K}_{2} \mathrm{Cr}_{2} \mathrm{O}_{7}$ oxidation and subsequent $\mathrm{Hg}$ reduction for 
1 detection. Multi-compounds solution used for method calibration revealed redox ligands

2 exchanges with $\mathrm{Hg}$, illustrating the difficulties of $\mathrm{Hg}$ speciation. More recently, reversed

3 phase HPLC was used after extraction with different nitric acid solutions and previous isotope

4 dilution mass spectrometry (IDMS) and speciated isotope dilution mass spectrometry $5 \quad($ SIDMS $)[18]$.

6 Until now, most of these approaches were used sequentially and no comparison between the 7 different protocols was made in order to attest accuracy of proposed speciations. Moreover, most of the proposed procedures are based on heavy equipment, unavailable on field or near production sources in order to speciate mercury of native gas condensates or crude oil. Small changes in procedure might have important impacts on final results, and this combined with

11 potential evolutions of $\mathrm{Hg}$ species over time will require serious validation steps for each 12 proposed method. Such a comparison based on model molecules validation, between a fractionation procedure and gel permeation chromatography coupled with ICP/MS is

14 proposed. This approach has two main advantages. On the one hand, the results are crosschecked with two procedures, and on the other hand, a procedure is possible on-site with little material allowing monitoring of $\mathrm{Hg}$ species evolution over time within sampled petroleum 17 cuts.

\section{EXPERIMENTAL SECTION}

\subsection{Solutions of model molecules}

21 Six synthetic feeds were considered to evaluate speciation methods. Each solution contained only one specific form of mercury. The species in these solutions were Elemental Mercury

$23\left(\mathrm{Hg}^{\circ}\right)$, Diphenyl Mercury, Dichloride Mercury, and three kinds of Mercury(II) Alkyl24 Thiolates, Pentyl, Octyl and Tetradecyl. 
For $\mathrm{Hg}^{\circ}$ solution, $0.5 \mathrm{~g}$ of pure $\mathrm{Hg}$ metallic (Ophram Saint-Fons France, triple distilled, 99.9999\%) was stirred for one week in a $500 \mathrm{ml}$ beaker filled with xylene previously purged with nitrogen. The solution obtained was collected discarding the bottom of the beaker containing elemental $\mathrm{Hg}$ and some xylene. This solution was measured for total $\mathrm{Hg}$ content and diluted furthermore to avoid precipitation of $\mathrm{Hg}$ due to temperature variation.

For the Diphenyl Mercury solution, standard was purchased from Sigma-Aldrich Co. at $>97 \%$ purity. For Dichloride Mercury solution, standard was purchased from Merck KGaA at $<99.5 \%$ purity. These compounds were solubilised directly by addition of xylene and stirred until complete dissolution.

For mercury (II) pentyl-thiolate, octyl and tetradecyl, these were synthetized according to

Steinfatt \& Hoffmann [19] with mercury (II) nitrate as the initial salt. The three product obtained were analyzed by elementary analysis $\left(\mathrm{C}, \mathrm{H}, \mathrm{S}\right.$ and $\mathrm{Hg}$ ) and also verified by ${ }^{1} \mathrm{H}$ and ${ }^{13} \mathrm{C}-\mathrm{NMR}$. Intermediate solution at $50 \mathrm{mg} / \mathrm{l}$ of $\mathrm{Hg}$ were prepared for further dilution. obtain a mercury concentration of approximately $100 \mu \mathrm{g} / \mathrm{L}$ in xylene. These solutions were measured for total $\mathrm{Hg}$ content.

Conostan ${ }^{\circledR} \mathrm{Hg}$ monoelemental standards in oil (1000 $\mathrm{mg} \mathrm{l}^{-1}$ ) (ConocoPhillips, Houston, TX) was used for instrument calibration control check.

\subsection{Sampling Bottle for crude oil and condensate collection}

For airtight conditions, commercial Swagelok cylinders (NZ7172 304L-HDF4-T) coated with Teflon internally were used to perform samplings directly on-site. Regular PTFE box and epoxy tank were also used to routinely collect liquid streams in aerobic conditions for onsite analysis. 


\section{$1 \quad$ 2.3. Crude oil, condensate and straight-run gasoline samples}

2 The crude oil, the straight-run gasoline and the two condensates were sampled with Swagelok

3 cylinders directly onsite. The Swagelock cylinders were pressurized with nitrogen in the

4 laboratory to evacuate any traces of oxygen prior to the sampling and were flushed on-site

5 with the crude oil or condensate for 3 minutes before sampling. Initial speciation and total $\mathrm{Hg}$

6 content were performed on site. Hydrocarbon liquid streams were then shipped back to IFPEn

7 facilities for further speciation using hyphenated techniques.

\subsection{Instrument for Mercury Quantification}

For total $\mathrm{Hg}$ analysis in organic substances and on site analysis, a PE-1000 (Nippon

Instrument Corporation, Tokyo, Japan) was used based on combustion followed by gold trapping and atomic fluorescence detection. Air and Argon were used as carrier gas alternatively. The mercury fluorescence was measured with an exciting wavelength of 253,7 nm. The system was equipped with an autosampler with syringe injection. The instrument was calibrated with solutions of diphenyl mercury in xylene and daily checked with Conostan mercury standard solution. The instrument was dedicated to the organic liquid samples with the exception of samples containing significant level of chlorine.

For aqueous solution containing cysteine or any others aqueous solutions, a DMA-80 (Milestone, Bergamo, Italy) was used with quartz vessel, combustion and atomic absorption detection. Oxygen was the carrier gas. The absorbance was measured at $253.7 \mathrm{~nm}$. The system was equipped with an autosampler with boat injection. The instrument was calibrated with aqueous standard solutions of mercury with $0.1 \%$ cysteine and daily checked with the aqueous standard solution. The instrument was dedicated to aqueous samples due to their good stability and low volatility. Analyses of organic liquid samples with a significant level of chlorine were nevertheless also carried out with the DMA-80. In this case, a special 
1 adsorbing material was added into the boat with the sample. This material absorbed the

2 sample matrix to reduce the volatility of the sample and to insure matrix decomposition in a 3 controlled fashion.

4 For $\mathrm{Hg}$ in vapour phase, gold sand trap were used and analyse with a SP-3D (Nippon 5 Instrument Corporation, Tokyo, Japan) based on heat-desorption with cold-vapor atomic 6 absorption spectrometry detection. The carrier gas was managed by a continuous flow of 7 purified dry air. The absorbance was measured at $253,7 \mathrm{~nm}$. The instrument was calibrated with a mercury calibration monitor (model NIC MB-1). This system generated a mercury vapour in a confined mercury reservoir controlled in temperature. This gas phase is saturated with mercury and is collected with a glass syringe. This aliquot is directly injected in the instrument through the gold amalgamator. At the same time, the sampling temperature is referred to an abacus to calculate the mercury concentration of the volume of vapour injected. A calibration check was daily performed.

Parallel to these analyzers, various sample (organic, aqueous, solid, gold sand trap) were also analyzed on a AMA254 Mercury Analyser (LECO Corporation, St. Joseph, Michigan, US). This apparatus involved combustion in oxygen followed by gold amalgamation with detection by atomic absorption. A continuous flow of oxygen carried the decomposition products through a catalyst bed. The absorbance was measured at $253.7 \mathrm{~nm}$. The instrument was calibrated with certified, mercury aqueous standard solution stabilised with $5 \mathrm{mg} / \mathrm{L}$ of 21 gold with $1 \%$ of nitric acid. The calibration of the instrument was used for all kind of sample and was daily checked with standard quality control solutions. The analyses of aqueous solution, hydrocarbon liquids and gold sand trap were performed with the AMA254 Mercury Analyser with a few changes of sample injection. 
The aqueous solutions were pipetted directly into a boat up to a maximum volume of 200

$2 \mu \mathrm{L}$. The loading of the liquid into the boat were carried out at the last possible moment just

3 before the boat entrance into furnace tube ensuring the stability of the sample.

4 The hydrocarbon liquids weren't loaded directly into the boat as they might decompose 5 explosively. This was prevented by using only a small volume $(<30 \mu \mathrm{L})$ of liquid 6 hydrocarbon, and by absorbing this onto a sample introduction adsorbing pellet placed in the 7 boat. The pellet was cleaned with a series of internal blanks' run before the sample analysis. 8 The loading of the liquid onto the sample introduction pellet was carried out, up to a 9 maximum volume of $20 \mu \mathrm{L}$, at the last possible moment just before the boat enters the furnace 10 tube

11 The volatile mercury was trapped by amalgamation with gold in a gold sand trap. These 12 gold sand traps were introduced directly in to the analyser using the gold sand trap head. Before the gold sand trap insertion into the head, a series of internal blanks was run to establish the background level.

For all these instrument (PE 1000, DMA-80, SP-3D, AMA 254), crosscheck tests were organized in order to validate equivalent response between the various instrument.

\subsection{Speciation by fractionation method}

Three kind of filter with three different porosity $0.5,2$ and $5 \mu \mathrm{m}$ were used for the analytical development. PTFE filter (PALL, Zefluor ${ }^{\mathrm{TM}}$ ) were purchased from VWR (Fontenay sous

21 Bois, France), paper filter (qualitative filter paper $n^{\circ} 410$ ) were also purchased from VWR 22 and stainless steel filter were obtained from Swagelock (Lyon, France) An aqueous solution containing $1 \% \mathrm{w} / \mathrm{w}$ of cysteine was required to perform the extraction of ionic forms of mercury during the fractionation speciation procedure. Cysteine standard 
1 was purchased from Nacalai Tesque Inc. at $>98 \%$ purity. Fresh solution was daily prepared by

2 dissolution of the standard in DI water in order to obtain a cysteine concentration of $1 \% \mathrm{w} / \mathrm{w}$.

\subsection{Speciation by SEC-ICP-MS Method} sector-field-ICP MS instrument (Thermo Fisher, Bremen, Germany). SEC-ICP/MS method was developed in a previous study [20] and further investigated with micro-columns [21-22]. SEC separation was achieved with a specific polystyrene-divinylbenzene columns designed for purpose by Shodex (porosity $150 \AA$, KF 402-1E). SEC method was performed at $30^{\circ} \mathrm{C}$ and ICP parameters were already given elsewhere [22]. Sulfur detection was obtained with ${ }^{32} \mathrm{~S}$ isotope reading with medium resolution and mercury determination was obtained by following ${ }^{202} \mathrm{Hg}$ and ${ }^{200} \mathrm{Hg}$ isotope. Mobile phase was THF with a flow rate of $13 \mu \mathrm{L} \cdot \mathrm{min}^{-1}$.

\section{ANALYTICAL STRATEGY FOR HG SPECIATION BY}

\section{FILTRATION, PURGE AND EXTRACTION}

The fractionation procedure based on UOP 938 is a conventional approach used to differentiate between mercury species including particulate mercury, volatile mercury, organic non-ionic mercury and ionic mercury. The method involves three major steps: a filtration, a gas purge and a liquid-liquid extraction. A global diagram is shown in Figure 1 to understand the global mercury speciation method. The total mercury is quantified in the total sample before the filtration step. The particulate mercury can be quantified by analysing the total mercury trapped on the filter paper after the filtration or by measuring the $\mathrm{Hg}$ present after the filtration step and comparing it to the initial total content of $\mathrm{Hg}$ before the filtration. 
1 analyse the gold sand trap afterward. Both phases in the liquid-liquid extraction step can be

2 analysed in order to determine the content of ionic and non-ionic mercury in the sample.

\section{3.1. Optimization and improvement of the filtration step}

4 The extraction of the particulate forms of mercury consists of a filtration procedure of the 5 sample. The UOP method 938 recommends filtering the sample by pressure filtration.

6 The analytical strategy investigated the optimization of this filtration step with the use of

7 different type of filtration. Vacuum filtration was compared to syringe filtration to maximise 8 the efficiency of the filtration step and minimizing plugging of the filter. For vacuum

9 filtration, a gold trap was inserted between the filtration device and the diaphragm pump (KnF Neuberger Laboport, Type : N820.3FT.18). The results, illustrated on Figure 2 with two different types of feed, shows clearly that vacuum filtration has to be avoided with elemental $\mathrm{Hg}$, which is partially lost in the gas phase during filtration. A similar conclusion was obtained when using softer vacuum system like water pump.

Different porosity and material of the filter paper were tested to optimize the method. A metallic filter was compared to a PTFE filter. And different porosity of filter was used from $5 \mu \mathrm{m}$ to $0,5 \mu \mathrm{m}$. The results, illustrated on Figure 3 with two different types of feed, shows clearly that for a solution of model molecule, the porosity and the type of filter does not change the amount of retained mercury on the filter. However, for industrial feed, the choice of the type of filter in terms of porosity and material have to be carefully defined as it can significantly change the amount of particulate mercury speciated by this fractionation method.

21 Similar result have been obtained when using different porosity with PTFE or paper filter. We recommend the use of PTFE filter when the laboratory equipment available can manage the quantification of particulate mercury trapped on the filter by filter digestion. However, the filtration of waxy industrial feed can be difficult with a PTFE filter, then in this case a metallic filter is recommended and warmed up for filtration. 
1 A microwave digestion of the filter or PTFE paper was also developed to analyse the

2 particulate mercury as an aqueous solution with the mercury analyser. The results, given as

3 supplementary information (Figure AS) with two different types of feed, shows clearly that

4 for a solution of model molecule, the method does not overestimate the particulate mercury.

5 Moreover, for a synthetic feed containing a large amount of particulate mercury, the method

6 allows the successful recovery of the mercury trapped on the filter.

7

\subsection{Optimization and improvement of the purge step}

The extraction of the volatile forms of mercury consists of a gas purge procedure of the filtered sample. Indeed, though pure mercury tends to have a very low volatility at ambient temperature, it proves to be extremely volatile when dissolved in hydrocarbon feeds, probably due to its very non-ideal behaviour when diluted in such compounds. The UOP method 938 recommends the purge of $50 \mathrm{~mL}$ of the filtered sample. The purge was performed in a gas wash bottle with a helium flow rate of $350 \mathrm{~mL} / \mathrm{min}$. The filtered hydrocarbon solution was cooled down 30 min into an ice bath prior to purge the volatile forms of mercury. This step minimizes the loss of volatile organic compounds of the hydrocarbon stream during the purge step. The gas purge was performed varying the time of purge and the system was maintained at low temperature with the ice bath.

The analytical strategy investigated the optimization of gas purge step by the use of different time of purge and type of gas. No difference was observed when the use of helium was replaced by nitrogen, allowing to reduce the analyse cost and improving the ability to perform the mercury speciation method directly on site, outside of a conventional laboratory. Different gas purge times were also tested in order to optimize the delay to purge all the elemental $\mathrm{Hg}$ present within various solutions. A straight run gasoline already described in Table 1 was spiked with elemental $\mathrm{Hg}$ and the purge was stopped after 10, 30 minutes, one and two hours. 
1 The $\mathrm{Hg}$ collected on the gold trap was measured in order to determine the percentage of

2 elemental $\mathrm{Hg}$ collected over time. The same experiment was performed with a xylene solution

3 containing elemental $\mathrm{Hg}$ and the release of $\mathrm{Hg}$ is illustrated in Figure 4. A gas purge time of 2

4 hours is efficient enough to extract more than $95 \%$ of volatile mercury.

5 Finally, a specific home-made unit with pressure filtration was built according to Figure 5.

6 The home-made unit was developed for the specific application of the mercury speciation in

7 hydrocarbon sample. The equipment was developed allowing direct filtration and gas purge in 8 the same way, avoiding sample contamination, oxidation and mercury loss between these two 9 steps.

\subsection{Optimization and improvement of the extraction step}

12 The extraction of the ionic forms of mercury consists of a liquid-liquid extraction with the organic sample and an aqueous ionic solution. The UOP method 938 recommends the use of $0.01 \% \mathrm{w} / \mathrm{w}$ sodium chloride solution in water with a volume ratio of $2: 5$ (sodium chloride solution : organic sample). This protocol also specifies a strong magnetic agitation for $15 \mathrm{~min}$ and $10 \mathrm{~min}$ of time settle before the phases' separation.

The analytical strategy investigated the optimization of this extraction step with a L-cysteine aqueous solution, instead of a sodium chloride aqueous solution. This choice was induced by the fact that some mercury analysers are affected by chlorine concentration in samples that may damage the catalyst used for conversion of total $\mathrm{Hg}$ to $\mathrm{Hg}^{\circ}$ before gold trapping. The

21 volume ratio used was 1:2 (L-cysteine solution : organic sample) and a series of several successive extractions was used to reach an efficient ionic mercury extraction step. The $\mathrm{pH}$ parameter ( $\mathrm{pH}$ from 4 to 10) was investigated to improve the method but no improvement was observed. The optimization strategy also investigated the effect of mixing time, the number of successive extractions and the L-cysteine concentration. Mercury thiolates were used as 
1 model molecule for ionic $\mathrm{Hg}$ as $\mathrm{Hg}$ is easily exchanging thiolates groups in various situation

$2 \quad[17,23]$. As the Figure 6 shows, efficient conditions for the extraction step were seen with 10

3 min of extraction for 3 successive extractions with a $1 \% \mathrm{w} / \mathrm{w}$ L-cysteine solution. The

4 increase of the Cysteine concentration does not show improvement of the method.

\section{ANALYTICAL STRATEGY FOR HG SPECIATION BY}

\section{GPC-ICP/MS}

8 As for the preceding speciation scheme, an important validation procedure was carried out for GPC-ICP/MS method. Initial calibration of the column was performed with S compounds that are available in a great variety of mass and species (sulfides, disulfides and mercaptans) and can be easily detected by ICP/HRMS with medium resolution. These $\mathrm{S}$ molecules were injected within the column individually and their retention time were collected and correlated to the molecular mass of the chosen molecules. Figure 7 is illustrating the result obtained with various $\mathrm{S}$ molecules with molecular mass comprised between 62 and 659 Da. Model molecules containing $\mathrm{Hg}$ and $\mathrm{S}$ were analyzed with the same procedure and their mass were estimated with the correlation given on Figure 7. Results given in Table 3 show that for $\mathrm{Hg}$ compounds containing less than $15 \mathrm{C}$ atoms, a higher estimated mass is obtained with the correlation. As most of the products used for calibration are based on $\mathrm{S}$ molecules, the presence of $\mathrm{Hg}$ with a mass of 202 is shifting the molecular mass to a higher number. For higher molecular weight compounds, the procedure validates the response and retention time

21 of the column (Table 3). 


\section{5. COMPARISON OF STRATEGY FOR REAL FEEDS}

2 Using the two procedures proposed and validated in the preceding chapter, different

3 petroleum product collected on site were speciated and their result were compared. Figure 8

4 and Table 2 illustrate the various information that was obtained with the two different

5 approaches. $\mathrm{Hg}$ speciation by filtration, purge and extraction was obtained on site directly

6 after sampling and speciation by GPC coupled to ICP/MS was done with sample brought back

7 to the laboratory in pressurized Swagelock cylinders. For the conventional approach based on

8 fractionation, the North Sea condensate revealed the presence of elemental mercury with a

9 contribution of $3 \%$ to the total $\mathrm{Hg}$ concentration. The presence of elemental mercury was not

10 observed with the GPC-ICP/MS chromatograph and this is coherent with the quantification

11 limit of elemental mercury at approximately $10 \mu \mathrm{g} / \mathrm{l}$ with GPC-ICP/MS. The level of

elemental $\mathrm{Hg}$ in the sample should be around $5 \mu \mathrm{g} / \mathrm{l}$ according to the speciation obtained by

13 purging the solution and this low concentration was then below the quantification limit of the

14 GPC-ICP/MS approach. Moreover, the speciation by GPC-ICP/MS was obtained after 3

15 weeks due to the shipping of the Swagelock cylinder to the laboratory. Even if the Swagelock cylinder should maintain anoxic conditions and preserve the oxidation of elemental mercury, such a small quantity might not be retained over such a long time. However, the GPCICP/MS approach did provide molecular size information of the ionic mercury that was detected after the cysteine extraction. In the case of this North Sea condensate, the molecule containing $\mathrm{Hg}$ was composed of a few carbon atoms with size comprised around $300 \mathrm{Da}$.

21 Complementary information was also obtained for the SE Asian crude oil and condensate. Speciation obtained by filtration, purge and extraction showed mainly the presence of ionic and non-ionic organic $\mathrm{Hg}$ for both products (Table 2). The presence of particulate $\mathrm{Hg}$ was also observed for the condensate and this was confirmed by analysing the filter used by SEM- 
1 Further information was obtained with the GPC-ICP/MS approach (Figure 8). For both SE

2 Asian products, the molecules containing $\mathrm{Hg}$ had a higher molecular weight than the North

3 Sea condensate. In this case, a broader distribution was observed with molecular weight

4 around 400 and $900 \mathrm{Da}$ for the SE Asian condensate and crude oil respectively. The

5 molecules associated with $\mathrm{Hg}$ were containing between 10 to 100 carbon atoms, showing a

6 broad diversity of molecules with high molecular weight. No elemental mercury was detected,

7 neither with the purge procedure, nor with the GPC-ICP/MS approach.

8 The two approaches proposed are complementary. Conventional approach based on filtration,

9 purge and extraction is giving physico-chemical information on the various chemical 10 categories of $\mathrm{Hg}$ contained in the samples. Structural information is obtained by GPC coupled 11 to ICP/MS by measuring specifically the size of $\mathrm{Hg}$ compounds. Particulate $\mathrm{Hg}$ is only 12 obtained with the filtration, whereas $\mathrm{Hg}^{\circ}$ can be measured with the purge and also detected by GPC-ICP/MS.

\section{RAPID EVOLUTION OF HG SPECIES OVER TIME FOR} CERTAIN LIGHT CUTS

In order to validate the air-tight sampling for various products in the refinery in Swagelock cylinders, two samples of straight-run gasoline were collected in Swagelock cylinders. As no mercury was detected in this sample, the gasoline sampled within the refinery was spiked with $70 \mathrm{ppb}$ of elemental $\mathrm{Hg}$ dissolved in purged xylene. One cylinder of this composite sample was partially opened and distributed in a beaker directly at the laboratory of the refinery to follow the evolution of the speciation. Directly after opening the bottle, the speciation was carried out with the filtration, purge and extraction procedure and this speciation was conducted again after 7 hours of exposure in the laboratory. Results are given 
1 precipitate was filtered and observed by SEM-EDS at IFPEN facilities. Agglomerates of

2 particles (supplementary information, Figure BS) were observed with high electron density.

3 The average size of the agglomerates was in the range of 5-10 $\mu \mathrm{m}$ and the individual particles

4 in the aggregates were approximately $1 \mu \mathrm{m}$ in diameter. Further analysis by EDS reveals

5 clearly the presence of $\mathrm{S}$ and $\mathrm{Hg}$ in these particles, probably due to the precipitation of $\mathrm{Hg}$

6 sulphide in the sample.

7 The second air-tight Swagelok cylinders containing the spiked gasoline were sent to IFPEn 8 facilities for further analysis. Mercury speciation was carried out with GPC-ICP/MS and the

9 presence of elemental $\mathrm{Hg}$ was confirmed as illustrated on Figure 8. S speciation was also obtained 3 weeks later and this analysis still reveals the presence of $\mathrm{H}_{2} \mathrm{~S}$ with a concentration of $14 \%$ of the total $\mathrm{S}$ content. This information is important as it proves the conservation of the anoxic conditions during the shipping of the sample. It is also important information to understand the rapid evolution of the elemental mercury to mercury sulphide as observed on the filter collected on site after $24 \mathrm{~h}$ of air exposure. These results validated the fact that $\mathrm{Hg}$ speciation can be obtained with GPC-ICP/MS even if the laboratory is not available on site.

\section{CONCLUSION}

Two different approaches used to speciate $\mathrm{Hg}$ in petroleum fractions were proposed and validated with model molecules. The validation of the filtration, purge and extraction procedure has shown that some steps are crucial in order to get reproducible results on the speciation. Typically for filtration, the result of the speciation is driven by the size of the filter, leading to a different result if the porosity of the filter is not respected. The purge step is less sensitive to change, the type of gas has no impact on the final result but a purge time 
1 between the two phases and the number of extractions is important to have a confident yield

2 of ionic $\mathrm{Hg}$.

3 Based on these validation, subtle variations in the parameters of the method can have drastic

4 changes in the final result and special care should be taken when speciation analysis are

5 conducted in different laboratories. Then crosschecking with an alternative method is

6 important and this was proposed in this article with gel permeation chromatography

7 hyphenated to ICP/MS.

8 These two approaches were validated with model molecules in order to verify the behaviour

9 of different types of $\mathrm{Hg}$ to the proposed scheme. These methods were also compared with real

10 product obtained from various locations in the world. Speciation carried out by filtration,

11 purge and extraction was done directly on site at the refinery and compared with GPC

12 hyphenated to ICP/MS after the samples have been brought back to the laboratory. Particular attention to the sampling procedure and the type of container used are then essential in order to maintain the speciation of the $\mathrm{Hg}$ between the time of sampling and time of analysis.

Physico-chemical information were obtained with the conventional approach based on filtration, purge and extraction and structural information was also obtained with GPCICP/MS. Comparison of speciation analysis done at different times shows the evolution of elemental mercury as it is exposed to air. Sampling needs then to be carefully carried out in order not to affect drastically the final information obtained by speciation.

The proposed procedure for sampling is also capable of maintaining the stability of the 21 species over more than 3 weeks when careful sampling is implemented.

\section{ACKNOWLEDGMENTS}

24 Catherine Bayle and Nathalie Texier from IFPEN are thanked for their help with the various $25 \mathrm{Hg}$ instrument. Caroline Aitken from Expro is thanked for her support during the field 
1 sampling. C. Arnaudguilhem and B. Bouyssiere (LCABIE-IPREM UMR 5254, CNRS /

2 UNIVERSITE DE PAU ET DES PAYS DE L'ADOUR) are thanked for all their advices on 3 ICP-HR/MS, which have helped to achieve this work. The financial support of IFPEn and

$4 \quad$ EXPRO for funding this study are greatly acknowledged 5 


\section{REFERENCES}

\section{UNEP report}

2. Programme des Nations Unies pour l'environnement. Rejets de mercure provenant de l'industrie du pétrole et du gaz 4 août 201115 pages

3. Bensefa-Colas, L.; Andujar, P.; Descatha, A. (2011) Mercury poisoning. Revue de Médecine Interne, 32, 416-24

4. Wilhelm S. M. (1999) Avoiding exposure to mercury during inspection and maintenance operations in oil and gas processing. Process Safety Progress, 18, 178-88

5. Kehal, M; Mennour, A; Reinert, L; Fuzellier, H (2004) Heavy metals in water of the Skikda Bay. Environmental Technol., 25, 1059-65

6. Wilhelm S. M. \& Bloom N. (2000) Mercury in petroleum. Fuel Processing Technology, 63, 1-27

7. Tao, H., T. Murakami, M. Tominaga, and A. Miyazaki (1998) Mercury speciation in natural gas condensate by gas chromatography-inductively coupled plasma mass spectrometry. J. Anal. At. Spectrom., 13(10), 1085-1093

8. Bouyssiere, B., F. Baco, L. Savary, R. Lobinski (2002) Speciation analysis for mercury in gas condensates by capillary gas chromatography with inductively coupled plasma mass spectrometric detection. J. Chromatogr. A, 976, 431-439

9. Snell, J.P., W. Frech, Y. Thomassen (1996) Performance improvements in the determination of mercury species in natural gas condensate using an on-line amalgamation trap or solid-phase micro-extraction with capillary gas chromatographymicrowave-induced plasma atomic emission spectrometry. Analyst, 121, 1055-1060

10. Shafawi, A; Ebdon, L; Foulkes, M; Stockwell, P; Corns, W (2000) Preliminary evaluation of adsorbent-based mercury removal systems for gas condensate. Analytica Chim Acta, 415, 21-32 
11. Bouyssiere, B., F. Baco, L. Savary, R. Lobinski (2000) Analytical methods for speciation of mercury in gas condensates: Critical assessment and recommendations. Oil Gas J., 55, 639-48

12. Suvarapu L.; Seo Y.-K.; Baek S.-O. (2013) Speciation and determination of mercury by various analytical techniques. Rev. Anal. Chem., 32(3), 225-45

13. Furuta, A., X. Sato, K. Takahashi (1990) Trace analysis of mercury compounds in natural gas condensate. Proc. of the International Trace Analysis Symposium

14. Bloom, N.S. (2000) Analysis and stability of mercury speciation in petroleum hydrocarbons. Fresenius. J. Anal. Chem., 366, 438-443

15. Frech, W., D.C. Baxter, B. Bakke, J. Snell, and Y. Thomassen (1996) Determination and speciation of mercury in natural gases and gas condensates. Anal. Commun., 33, H7-H9

16. Pontes F.V.M., CarneiroM.C., Vaitsman D.S., Monteiro M.I.C, Neto A.A., Tristão M.L.B (2014) Investigation of the Grignard reaction and experimental conditions for the determination of inorganic mercury and methylmercury in crude oils by GC-ICPMS. Fuel, 116, 421-6

17. Schickling, C. and J.A.C. Broekaert (1995) Determination of mercury species in gascondensates by online coupled high performance liquid chromatography and coldvapor atomic absorption spectrometry. Appl. Organomet. Chem., 9, 29-36

18. Rahman, G.M.M.; Fahrenholz, T.M.; Kingston, H.M.S.; Pamuku, M.; Hwang, J.D.; Young, L.A. (2010) Speciation of Mercury in Crude Oil Using Speciated Isotope Dilution Mass Spectrometry. Spectroscopy, 25, 36-41

19. Steinfatt I., Hoffmann G. (1994) The Redox Behaviour of Elemental Mercury and Diorganyl Disulphides. Z. Naturforsch. 49b, 1507-10 
20. Caumette G., Lienemann C.-P. , Merdrignac I., Bouyssiere B., Lobinski R. (2010) Fractionation and speciation of nickel and vanadium in crude oils by size exclusion chromatography-ICP MS and normal phase HPLC-ICP MS. J. Anal. At. Spectrom., $25,1123-112$

21. Pohl P., Dural D., Vorapalawut N., Merdrignac I, Lienemann C.-P., Carrier H., Grassl B., Bouyssiere B., Lobinski R. (2010) Multielement molecular size fractionation in crude oil and oil residue by size exclusion microchromatography with high resolution inductively coupled plasma mass spectrometric detection (HR ICP MS), J. Anal At. Spectrom., 25, (2010), 1974-7

22. Chainet F., Le Meur L., Lienemann C.-P., Courtiade M., Ponthus J.,Brunet-Errard L., Donard O.F.X (2012) Degradation processes of polydimethylsiloxane under thermal cracking conditions of hydrocarbons in an experimental pilot plant followed by size exclusion chromatography coupled to inductively coupled plasma high resolution mass spectrometry, Fuel Processing Technol. 104, 300-9

23. Bach R. D., Rajan S.J., Vardhan H.B., Lang T.J., Albrecht N.G. (1981) ${ }^{1}$ H NMR rate constants and mercury-199 FT NMT equilibrium constants involved in disulfide cleavage by methylmercury, J. Am. Chem. Soc. 103, 7727-34 


\section{TABLES}

\begin{tabular}{|c|c|c|c|c|}
\hline & $\begin{array}{c}\text { SE Asian } \\
\text { Crude }\end{array}$ & SE Asian & North Sea & Straight-run \\
& oil & Condensate & Condensate & gasoline \\
\hline total Hg (ppb) & 500 & 180 & 165 & $<1$ \\
\hline S concentration & 686 & 163 & 103 & 41 \\
\hline IBP $\left[{ }^{\circ} \mathbf{C}\right]$ & 50 & 35 & & 15 \\
\hline FBP $\left[{ }^{\circ} \mathbf{C}\right]$ & 653 & 473 & 410 & 171 \\
\hline
\end{tabular}

Table 1: Main properties of the 4 feed used for the validation of the speciation scheme

\begin{tabular}{|c|c|c|c|c|}
\hline $\begin{array}{c}\text { speciation of the } \\
\text { solution (\% of }\end{array}$ & SE Asian & SE Asian & North Sea & $\mathbf{H g}^{\circ}$-doped \\
Hg $_{\text {tot }}$ ) & oil & Condensate & Condensate & gasoline \\
\hline total Hg (ppb) & 500 & 180 & 165 & 715 \\
\hline particulate Hg & 0 & 38 & 0 & 2 \\
\hline volatile Hg & 0 & 0 & 3 & 98 \\
\hline ionic Hg & 77 & 58 & 91 & 0 \\
\hline non-ionic organic & 23 & 4 & 5 & 0 \\
\hline
\end{tabular}

6 Table 2: Speciation obtained with the conventional approach on three different feed and a synthetic $\mathrm{Hg}^{\circ}$ solution. 


\begin{tabular}{|l|l|l|l|}
\hline Chemical Formulae & Theoretical mass & Estimated mass & Error \\
& {$[\mathrm{Da}]$} & according to equation & \\
\hline $\mathrm{C}_{2} \mathrm{H}_{6} \mathrm{~S}$ & 62 & 59 & $-5 \%$ \\
\hline $\mathrm{C}_{8} \mathrm{H}_{18} \mathrm{~S}$ & 146 & 160 & $10 \%$ \\
\hline $\mathrm{C}_{8} \mathrm{H}_{18} \mathrm{~S}_{2}$ & 178 & 162 & $-9 \%$ \\
\hline $\mathrm{C}_{14} \mathrm{H}_{30} \mathrm{~S}$ & 230 & 248 & $8 \%$ \\
\hline $\mathrm{HgC}_{28} \mathrm{~S}_{2} \mathrm{H}_{58}$ & 659 & 638 & $-3 \%$ \\
\hline $\mathrm{HgC}_{10} \mathrm{~S}_{2} \mathrm{H}_{22}$ & 407 & 293 & $-28 \%$ \\
\hline $\mathrm{HgC}_{16} \mathrm{~S}_{2} \mathrm{H}_{31}$ & 491 & 447 & $-9 \%$ \\
\hline
\end{tabular}

1 Table 3: Estimated mass of various compounds obtained according to correlation of Figure 7 compared to 2 theoretical value.

\begin{tabular}{|l|c|c|}
\hline & $\begin{array}{c}\text { Initial sample at } \\
\text { the sampling site }\end{array}$ & $\begin{array}{c}\text { Sample after 7 } \\
\text { hours }\end{array}$ \\
\hline Volatile $\mathrm{Hg} \mathrm{( \% )}$ & 87 & 9,4 \\
\hline Particulate $\mathrm{Hg}(\%)$ & 11 & 85 \\
\hline Extractible $\mathrm{Hg}(\%)$ & 1,3 & $<1$ \\
\hline Non extractible $\mathrm{Hg}(\%)$ & $<1$ & 6,1 \\
\hline
\end{tabular}

5

6 Table 3: Speciation obtained with the filtration, purge and extraction method on a straight-run gasoline 7 spiked with $\mathbf{H g}^{\circ}$.

8 


\section{FIGURES}

4

5

8

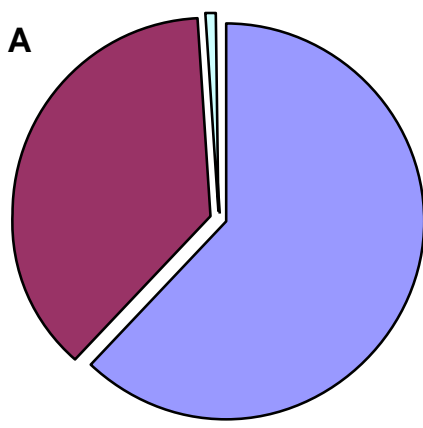

r lost on gold trap

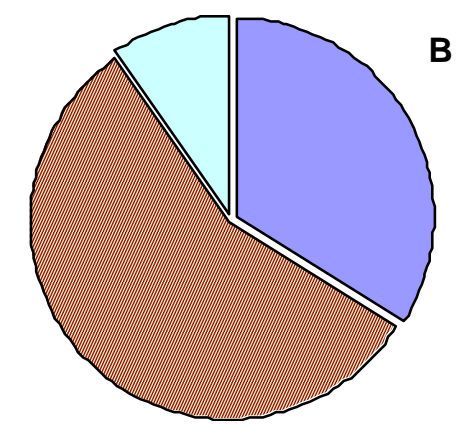

$\square$ deposited on filter $\square$ missing $\mathrm{Hg}$

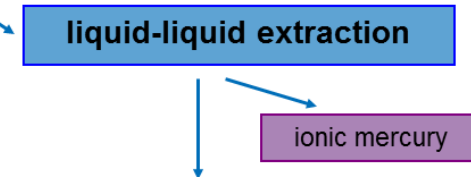

organic non-ionic mercury

Figure 1: General Diagram of the mercury speciation procedure based on UOP 938

Figure 2: Results obtained with vacuum filtration with the $\mathrm{SE}$ Asian Condensate(A) and the $\mathrm{Hg}^{\circ}$ doped xylene (B)

Figure 3 : Mercury in filtrate obtained with the SE Asian crude oil and the Pentyl-thiolate mercury(II) doped 


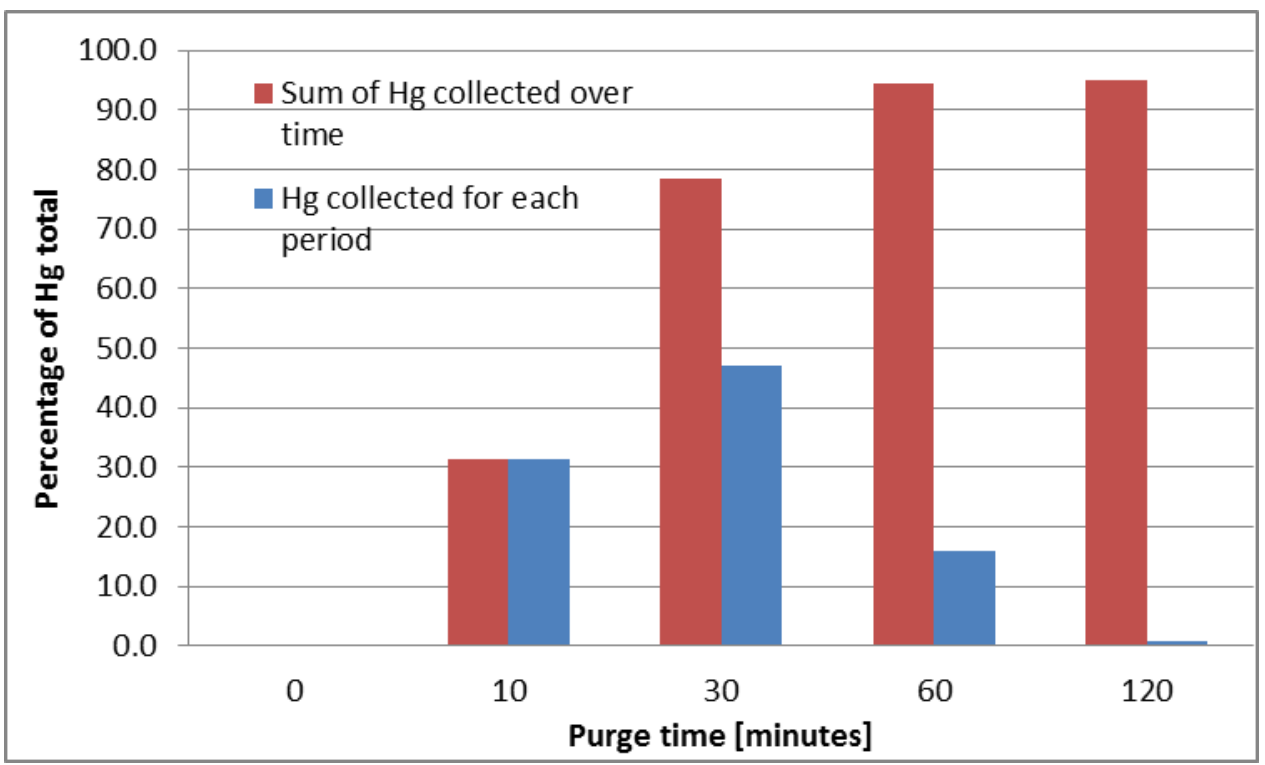

Figure 4: Optimization of the purge step : Sum and individual Hg collected over time from various solutions

containing $\mathrm{Hg}^{\circ}$

6

7

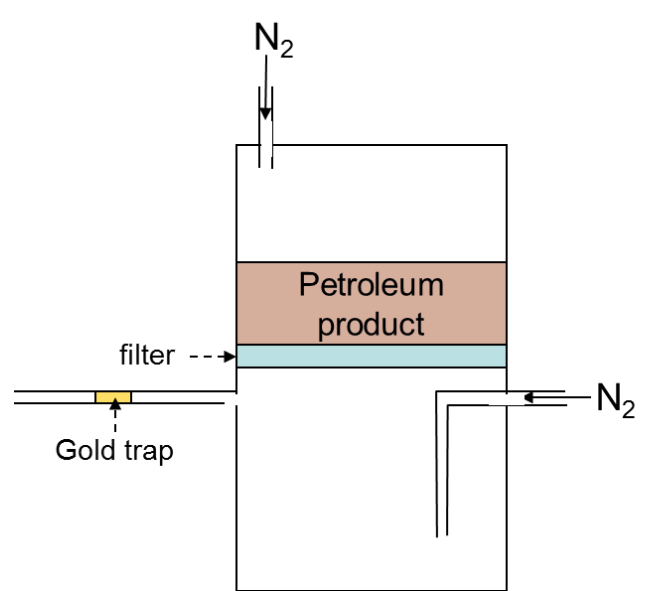

Step 1 : Filtration

$\rightarrow \mathrm{Hg}_{\text {particulate }}+\mathrm{Hg}_{\text {soluble }}$

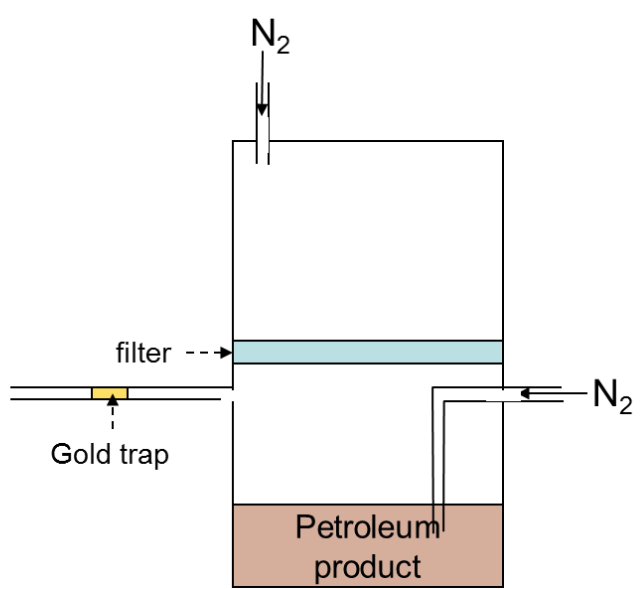

Step 2 : Purge

$\rightarrow \mathrm{Hg}_{\text {volatile }}$ on Gold trap

Figure 5: Scheme of the filtration unit 


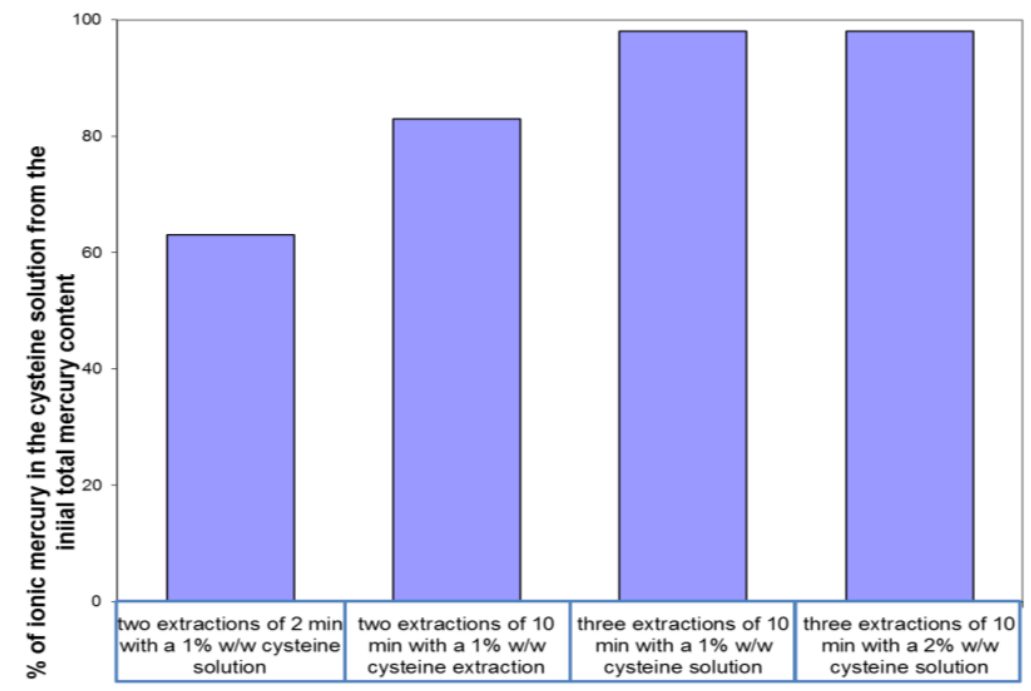

Figure 6: Optimization of ionic mercury species extraction : percentage of ionic mercury found in the

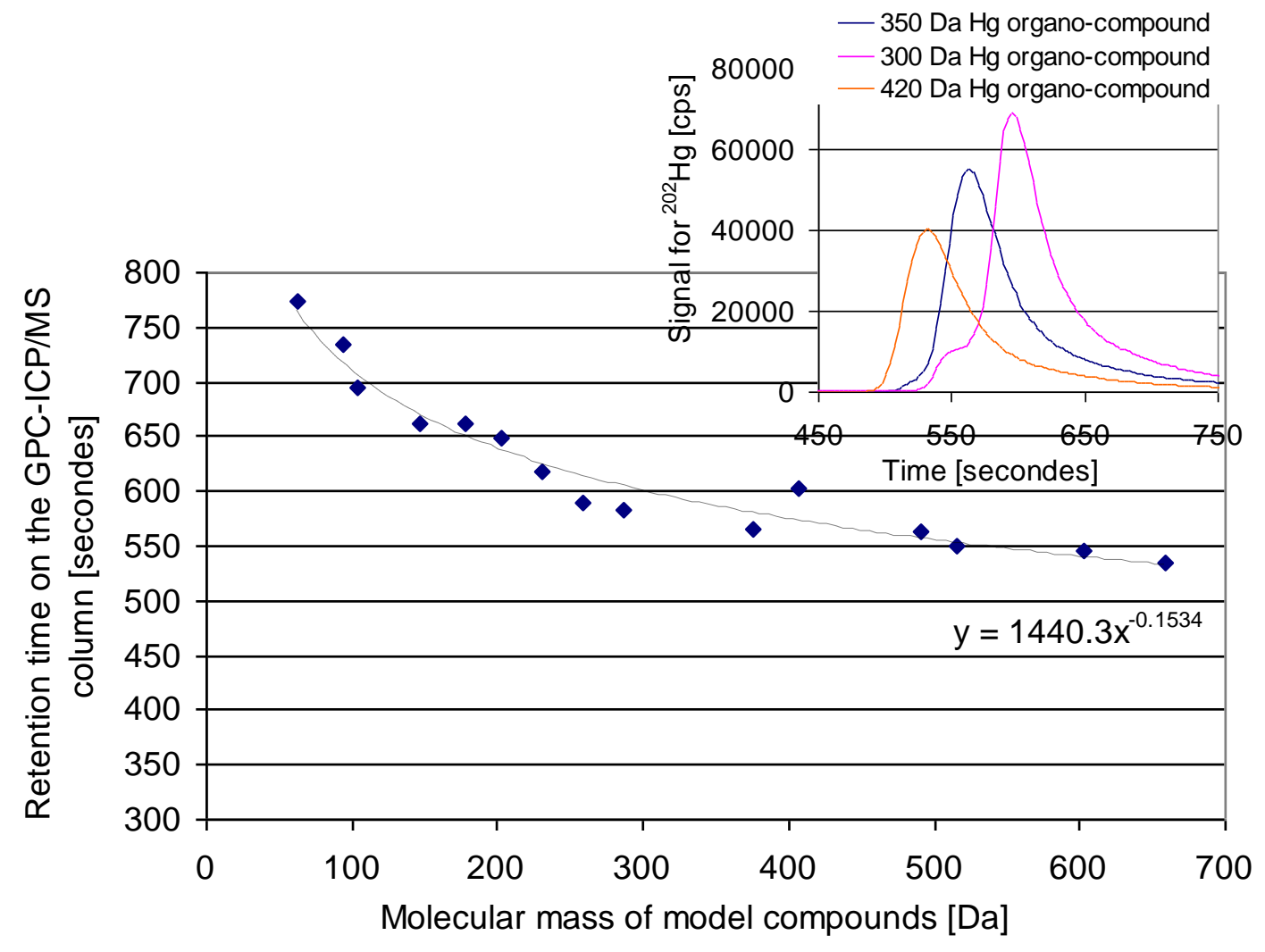

$6 \quad$ Figure 7: Calibration curve obtained with various S and Hg compounds with molecular mass comprised 


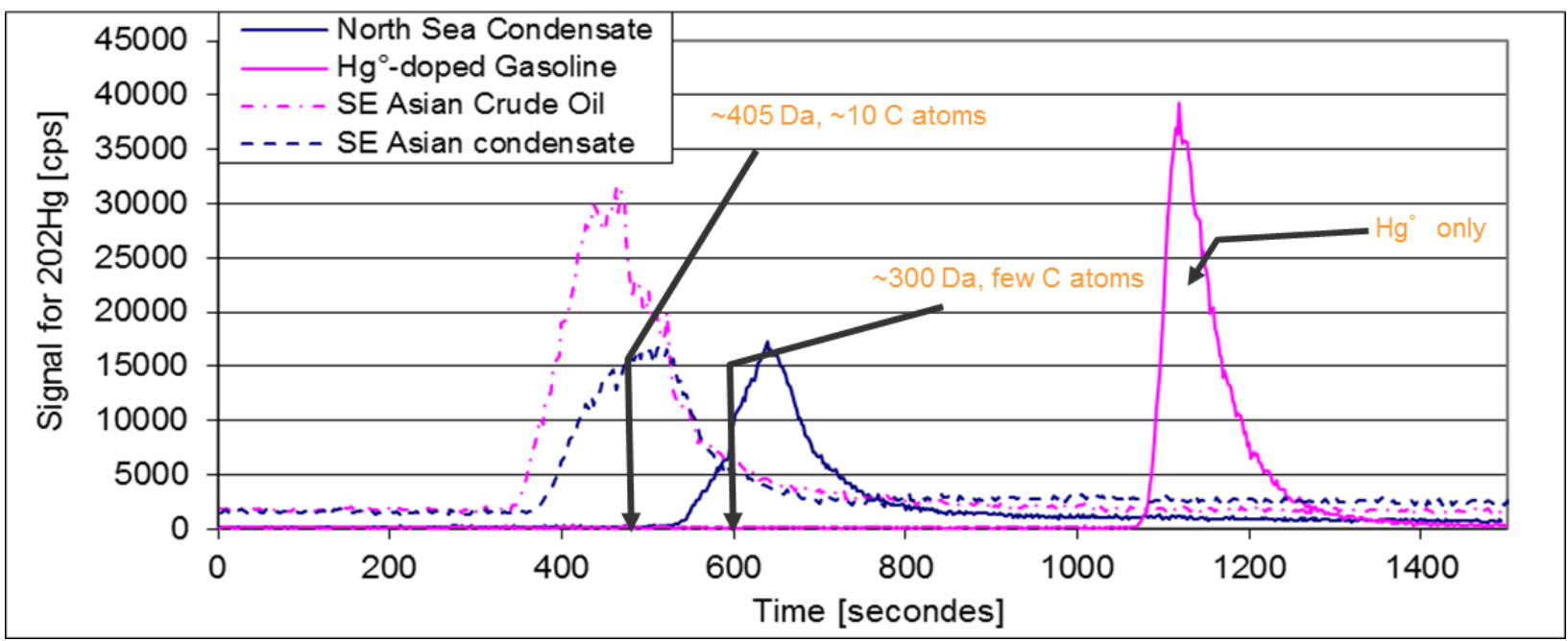

$4 \quad$ Figure 8: GPC-ICP/MS ${ }^{202} \mathrm{Hg}$ profiles obtained with three different feed and a straight-run gasoline spiked 5 with $\mathrm{Hg}^{\circ}$ solution.

6 


\section{Supplementary information :}

A

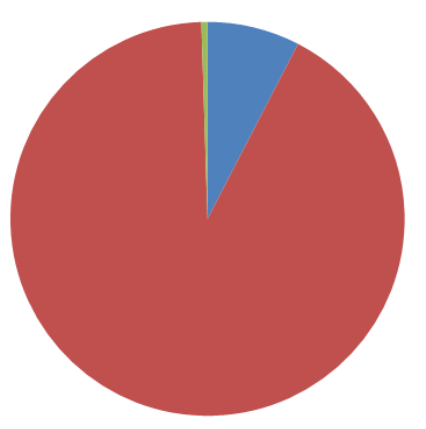

B

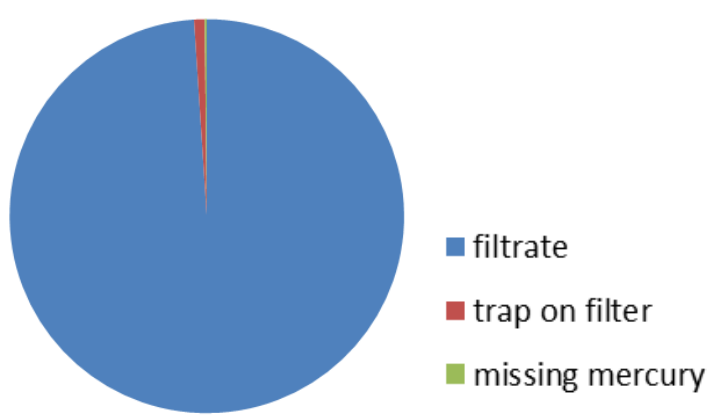

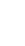

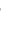

Figure AS: Results obtained on the filtration with the filter digestion procedure with the SE Asian Condensate (A) and the Pentyl-thiolate Mercury(II) doped xylene (B)

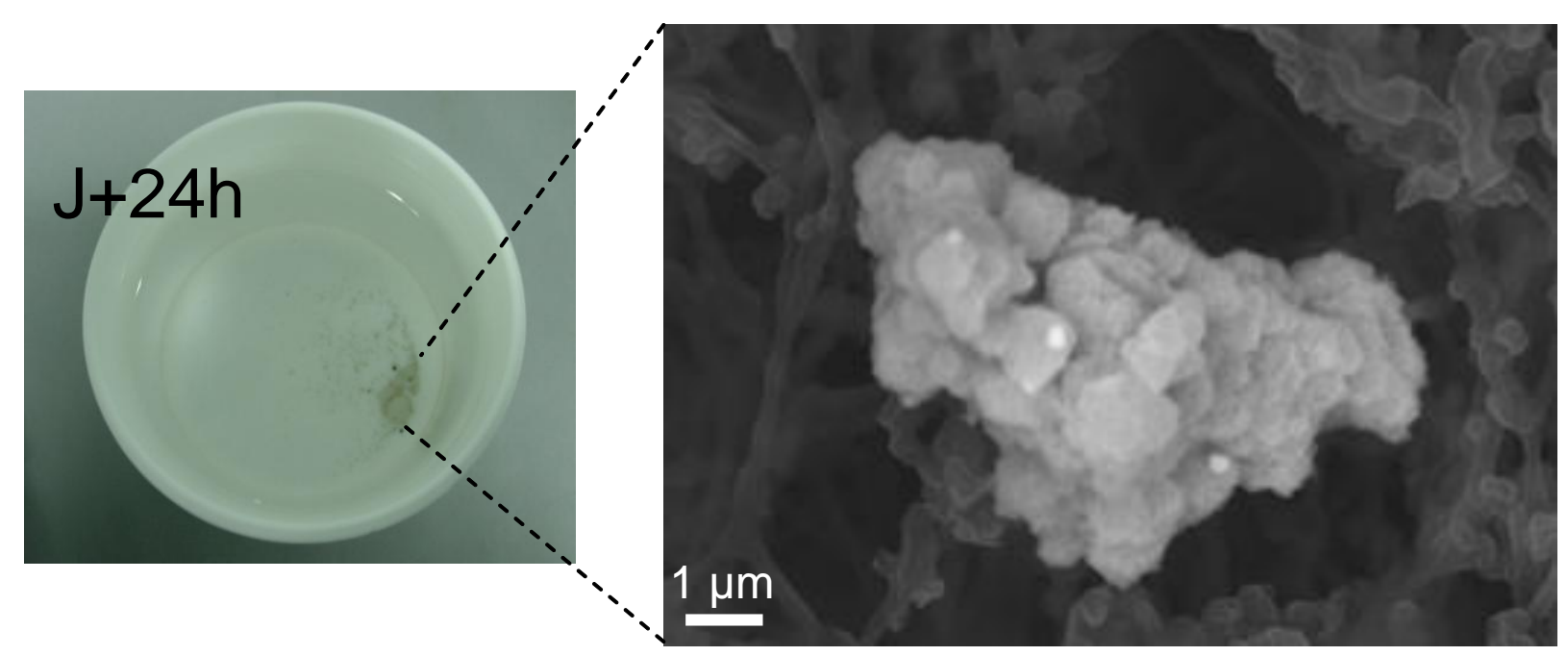

Figure BS: Typical HgS agglomerate obtained after 24 hours of conservation with air 


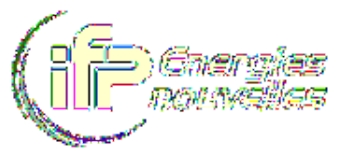

\section{Dr Aimaro Sanna \\ Fuel processing Technology}

Physic and Analysis division

Fax : +33478022020

charles.lienemann@ifpen.fr

March $30^{\text {th }}, 2014$

\section{Dear Dr Sanna,}

Please find enclosed the article entitled: Mercury speciation in liquid petroleum product: Various analytical approaches and comparison, by Florine Gaulier, Alexandre Gibert, David Walls, Michael Langford, Stuart Baker, Arnaud Baudot, Fabien Porcheron and myself. Please accept it as a candidate for publication in Fuel Processing Technology.

This paper deals with the specific subject of speciation of $\mathrm{Hg}$ in liquid petroleum product, a subject already discussed within your journal by many different authors. The proposed articles is proposing two different approaches to provide accurate speciation of $\mathrm{Hg}$ in liquid fuels. A solid validation of the different approaches are proposed and comparison of the two speciation schemes are performed with real hydrocarbon feeds produced in various locations of the world. Important results for the understanding of the behaviour of $\mathrm{Hg}$ in liquid organic streams and also recommendations for sampling and analysis are proposed. Thus, we believe that this paper drastically improves the general knowledge of $\mathrm{Hg}$ speciation and could be published in Fuel Processing Technology.

Kind regards,

\section{Charles Philippe Lienemann}


- Two different approaches used to speciate $\mathrm{Hg}$ in petroleum fractions were proposed and validated with various samples

- Variations in the parameters of the method can have drastic changes in the final result and special care should be taken when speciation analysis are conducted in different laboratories

- Physico-chemical information and structural information were obtained with the two approaches proposed and are complementary 